ARTICLE

https://doi.org/10.1038/s41467-020-14476-w

\title{
Vibronic coherence contributes to photocurrent generation in organic semiconductor heterojunction diodes
}

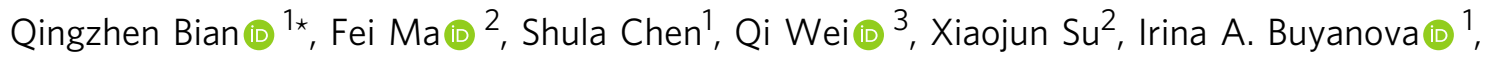
Weimin M. Chen (10 ${ }^{1}$, Carlito S. Ponseca Jr ${ }^{1}$, Mathieu Linares ${ }^{4}$, Khadga J. Karki ${ }^{2}$, Arkady Yartsev $^{2} \&$ Olle Inganäs ${ }^{1 \star}$

Charge separation dynamics after the absorption of a photon is a fundamental process relevant both for photosynthetic reaction centers and artificial solar conversion devices. It has been proposed that quantum coherence plays a role in the formation of charge carriers in organic photovoltaics, but experimental proofs have been lacking. Here we report experimental evidence of coherence in the charge separation process in organic donor/acceptor heterojunctions, in the form of low frequency oscillatory signature in the kinetics of the transient absorption and nonlinear two-dimensional photocurrent spectroscopy. The coherence plays a decisive role in the initial 200 femtoseconds as we observe distinct experimental signatures of coherent photocurrent generation. This coherent process breaks the energy barrier limitation for charge formation, thus competing with excitation energy transfer. The physics may inspire the design of new photovoltaic materials with high device performance, which explore the quantum effects in the next-generation optoelectronic applications.

\footnotetext{
${ }^{1}$ Department of Physics, Chemistry and Biology (IFM), Linköping University, 58183 Linköping, Sweden. ${ }^{2}$ Division of Chemical Physics, Lund University, 22100 Lund, Sweden. ${ }^{3}$ Institute of Applied Physics and Materials Engineering, University of Macau, Macau SAR, China. ${ }^{4}$ Department of Theoretical Chemistry and Biology, KTH Royal Institute of Technology, 10691 Stockholm, Sweden. *email: qingzhen.bian@liu.se; olle.inganas@liu.se
} 
n organic photovoltaics (OPVs), where the active layer consists of a nanostructured blend of donor (D) and acceptor (A) semiconductors, the key photophysical process is the generation of free charge carriers. Despite extensive studies indicating that the charge transfer (CT) state determines the photovoltage of OPVs, there are reports on the absence of this state in some efficient OPV material systems, claiming that long-range charge separation may occur directly from excitons generated within the polymer domain ${ }^{1,2}$. Intra-molecular or inter-molecular delocalization of charge may result in a decreased Coulomb binding 3,4 , allowing excitons (EX) to access more delocalized charge separated (CS) states, which are postulated to be the main precursors of free charge carriers ${ }^{5,6}$. Time-resolved experiments have found evidence for an ultrafast mobile charge generation on a sub-100 fs time scale $\mathrm{e}^{7,8}$.

Based on recent results, it has been proposed that coherent vibrational motion of the donor and/or fullerene may contribute to the primary step of the photoinduced charge-separation process $^{9,10}$. Other studies demonstrate measurement results that suggest coherences are too short lived to contribute significantly ${ }^{11}$. Nevertheless, the presence of other ultrafast processes (coherent intrachain energy transfer etc.) can contribute to the charge separation in the nanoscale systems ${ }^{12-14}$. However, a definitive correlation of oscillatory signals at femtosecond timescale to the charge separation and photocurrent generation is still lacking. Furthermore, the fundamental question about the structural factors that could be used to enhance a coherence contribution in photocurrent generation processes remains unanswered.

Here, we have studied ternary organic photovoltaic blends, with two polymer donors (D1, D2) and one fullerene (A1) or non-fullerene acceptor. Significantly enhanced photocurrent and fill factor of ternary devices have been observed, which cannot be explained by optical absorption and electronic transport properties. We have used transient spectroscopy techniques to unravel the materials' electronic structure, dynamics, and their contribution to the functionality of the devices. Insights on the timescale and processes leading to the formation of mobile charge carriers can be obtained using ultrafast transient absorption spectroscopy (TA) with sub-50 femtosecond (fs) temporal resolution, complemented by transient photoluminescence, transient photoconductivity and transient two-dimensional photocurrent spectroscopy (2DPS). Both films and working devices have been investigated to confirm the consistency of the transient behavior. We have found an oscillatory transient feature responsible for the ultrafast charge separation after the primary excitation, and the frequency of oscillation encompasses a number of low frequency vibrational modes of one of the donor units. Two of the modes at frequencies 360 and $200 \mathrm{~cm}^{-1}$ features prominently in the transients indicating their importance in the ultrafast charge separation after the primary excitations. Our 2D photocurrent results clearly demonstrates a distinctive coherent photocurrent generation that persists for the initial $\sim 200 \mathrm{fs}$ in a high performance device, indicating that the vibronic (electronic-vibrational) coherences directly contributes to the formation of charge carriers and enhancement in the photocurrent in the device.

\section{Results}

Ultrafast charge separation. We focus on two optimized ternary blends (Ternary (L), D1:D2(L):A1 = 9:1:10, D2 with low molecular weight; Ternary $(\mathbf{H}), D 1: D 2(\mathrm{H}): A 1=7: 3: 10, D 2$ with high molecular weight) and two reference binary blends (D1:A1, D2: A1) (Supplementary Fig. 1). Figure 1a shows their molecular structures and Fig. $1 \mathrm{~b}$ is the steady and transient state absorption spectra of materials. The energy transfer between two donors in the ternary blend demonstrates enhanced excited state population (Supplementary Fig. 2). Furthermore, high molecular weight increases planar conformation and polaron delocalization ${ }^{15}$. Both of these advantages increase the possibility to explore the primary excited states dynamics (Fig. 1c).

We have used near-infrared probe pulses to study the spectral evolution of transient species (Supplementary Fig. 3). In this spectral range, the TA signal consists of the excited state absorption (ESA), stimulated emission (SE), and photo-induced absorption (PIA) of the charged species. Specifically, the PIA band of excited singlet $(1500 \mathrm{~nm})$ and charge carriers $(1140 \mathrm{~nm})$ can be clarified in the extended infrared region (Supplementary Fig. 4). The infrared kinetics (Fig. 2a) demonstrate that the $860 \mathrm{~nm}$ PIA band, increases simultaneously with the singlet exciton dissociation in the initial picoseconds (ps), indicating that the ultrafast charge transfer occurs between the singlet exciton states and the $860 \mathrm{~nm}$ PIA band species. Thus, we focus on the $860 \mathrm{~nm}$ signal, whose kinetics predominantly reflects charge transfer excitons, and provide a clear measurement of charge carrier generation from initially photogenerated excitons on the polymer. To follow the ultrafast charge dynamics, singlewavelength kinetic measurements have been employed with improved time-resolution and signal-to-noise level, using excitation and probe wavelengths at 550 and $860 \mathrm{~nm}$, respectively. For the blends (Fig. 2b), the kinetic curve at early times is dominated by ESA and later converts into the PIA of charged species. In the reference binary blends, the PIA signal reaches the maximum at $\sim 25 \mathrm{ps}$ for $D 2: A 1$ and $\sim 10 \mathrm{ps}$ for $D 1: A 1$. Furthermore, the PIA signal in $D 1: A 1$ is much stronger than that in $D 2: A 1$. In both ternary blends, the PIA signal reaches a

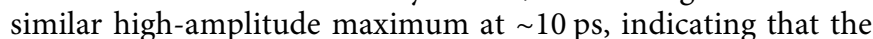
PIA signal in the ternary blends is mainly due to the positively charged species on the $D 1$.

In contrast to the binary blends, the kinetics of the ternary blends exhibit a fast decay ( $\sim 50 \mathrm{fs}$ ) of the initially-formed ESA. Furthermore, this component is much more pronounced in the Ternary $(\mathrm{H})$ blend. This short timescale $(\sim 100 \mathrm{fs})$ is consistent with the recently resolved ultrafast charge motion $(\sim 150 \mathrm{fs})$ at the donor/acceptor interface ${ }^{16}$, and it is attributed to rapid quenching of the excited states due to an ultrafast charge separation (CS) process (Fig. 1c). Particularly, this ultrafast charge separation is nearly temperature independent (Supplementary Fig. 5; Supplementary Table 1). It has been proposed that hybridization of donor and acceptor states can reduce the free energy barrier, making the excited state dissociate in an adiabatic process ${ }^{17}$. Such ultrafast charge separation reduces the Coulomb interaction between the electron-hole pairs and helps in the generation of the free charges. For the Ternary $(\mathrm{H})$ blend (Fig. 2a), there is an ultrafast charge carrier $(1140 \mathrm{~nm})$ rise kinetics in the initial $0.2 \mathrm{ps}$. Furthermore, the difference in the kinetics between the singlet and charge carrier in the initial rise can be traced after $100 \mathrm{fs}$. This timescale is consistent with the resolved charge separation process (Fig. 2b), indicating the prevalence of efficient charge generation in the Ternary $(\mathrm{H})$ blend due to the ultrafast $100 \mathrm{fs}$ kinetics. Moreover, compared to $D 1: A 1$ blend, the dynamics of charge carriers demonstrates a much faster generation after $100 \mathrm{fs}$ for the Ternary (H) blend (Supplementary Fig. 7). Furthermore, an almost similar charge carrier dynamics was observed after the initial 0.5 ps for the Ternary $(\mathrm{H})$ and the $D 1: A 1$ blend. These results further confirm that efficient charge generation in the Ternary $(\mathrm{H})$ blend results from the ultrafast charge separation process. To confirm the consistency of the charge separation in the transients, we investigate the same kinetics in the working OPV diodes under reverse bias. When free charge carriers are extracted by the reverse field, the number of charge carriers decreases and so does the subsequent PIA amplitude. As 
a

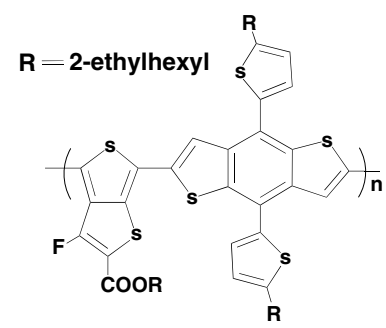

D1

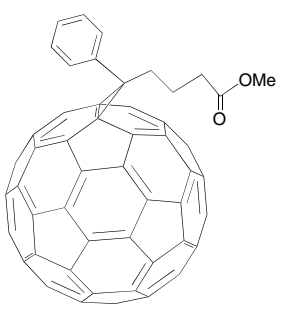

A1

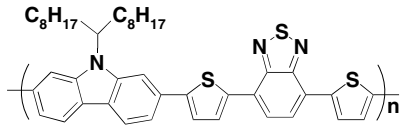

D2 b
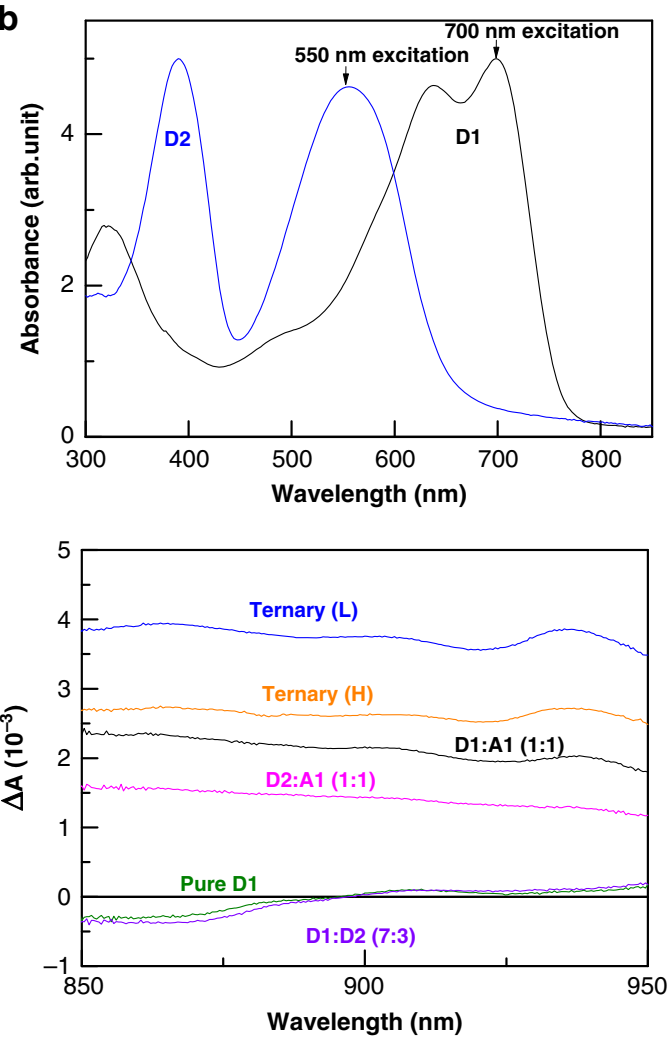

Wavelength (nm)

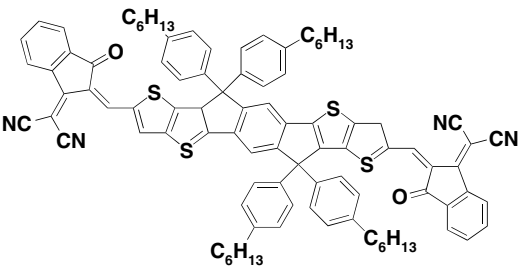

A2

c
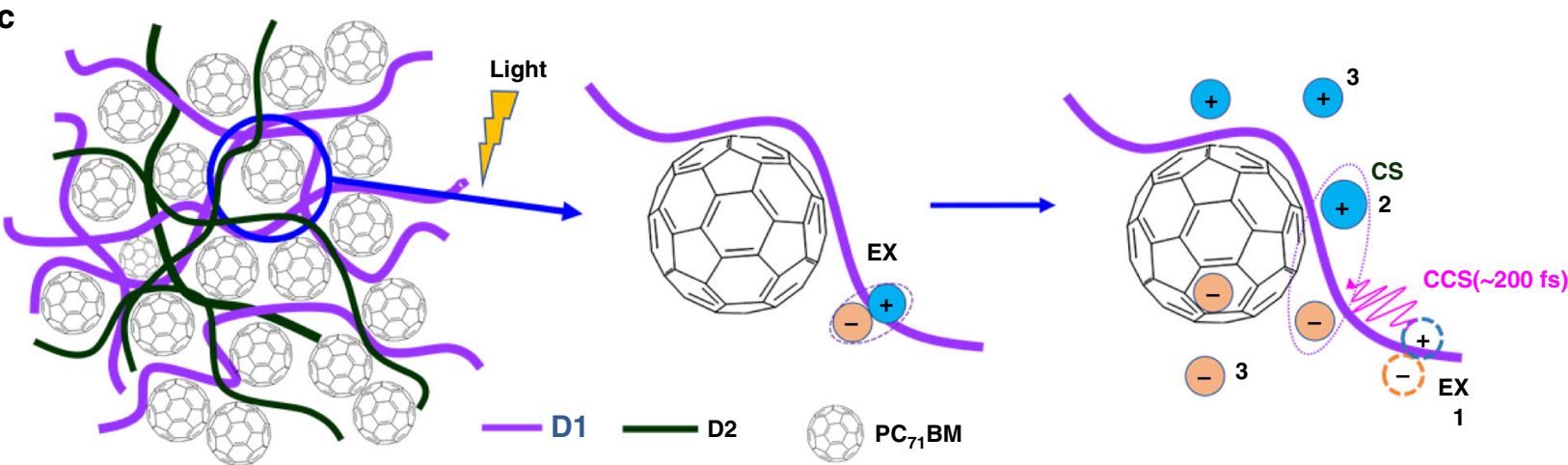

Fig. 1 Molecular structures and schematics of interfacial photophysical processes. a Molecular structures used in this study. $\mathbf{b}$ UV-Vis absorption for PCDTBT (D2, blue solid) and PTB7-th (D1, black solid); Transient absorption signal ( $10 \mathrm{ps})$ from the investigated pristine and blend films, pumped at $550 \mathrm{~nm}$. c Simple cartoon illustration of the charge photo-generation at a heterojunction. Light absorption generates excitons (EX) at the interface sites (1), then undergo coherent charge separation (CCS, persist in first $200 \mathrm{fs}$ ) into loosely bound electron-hole pairs ((2), charge separated states (CS)). The electron and hole separate further and form free charges (3).

expected, the Ternary $(\mathrm{H})$ device demonstrates a significant bias dependence in the initial $\sim 100 \mathrm{fs}$ timescale (Fig. 2c). In contrast, this feature is not observed in the $D 1: A 1$ device (Supplementary Fig. 8).

The rapid exciton dissociation and efficient charge generation in the Ternary $(\mathrm{H})$ blend can also be confirmed by other transient results. Time-resolved photoluminescence (TRPL) is sensitive to the singlet exciton dynamics. In these measurements, we have used the excitation at $445 \mathrm{~nm}$, close to that for the TA measurements (550 nm excitation). The results show that the excited states in the Ternary $(\mathrm{H})$ blend quenches faster (instrument limited) and more completely compared to the other two blends, where PL decay is slower and exhibits a long-lived visible tail up to hundreds of picoseconds (Supplementary Fig. 9). The TRPL measurements exhibit characteristic features of pulselimited population of the excited state followed by its depopulation in picoseconds in qualitative agreement with the TA dynamics (Supplementary Figs. 2, 9).

Ultrafast charge separation in the efficient Ternary $(\mathrm{H})$ can be further confirmed by monitoring photoconductivity using timeresolved terahertz (THz) spectroscopy (TRTS). Photoconductivity is a direct measure of the product of charge concentration and mobility brought about by the pump excitation ${ }^{18}$. For all the investigated films here, the same TRTS setup with a time resolution $>100 \mathrm{fs}$ and identical experimental conditions have been used. The change in photoconductivity per photon absorbed by the $D 1: A 1$ and Ternary $(\mathrm{H})$ blends excited at $400 \mathrm{~nm}$ as a function of pump-probe delay is shown in Fig. 2d. Both $D 1: A 1$ and Ternary $(\mathrm{H})$ blend exhibit an instrument limited rise time, while for D2:A1 no photoconductivity is obtained. The absence of the photoconductivity signal in $D 2: A 1$, despite the TA results showing charge generation before 100 ps (Fig. 2b), implies that 

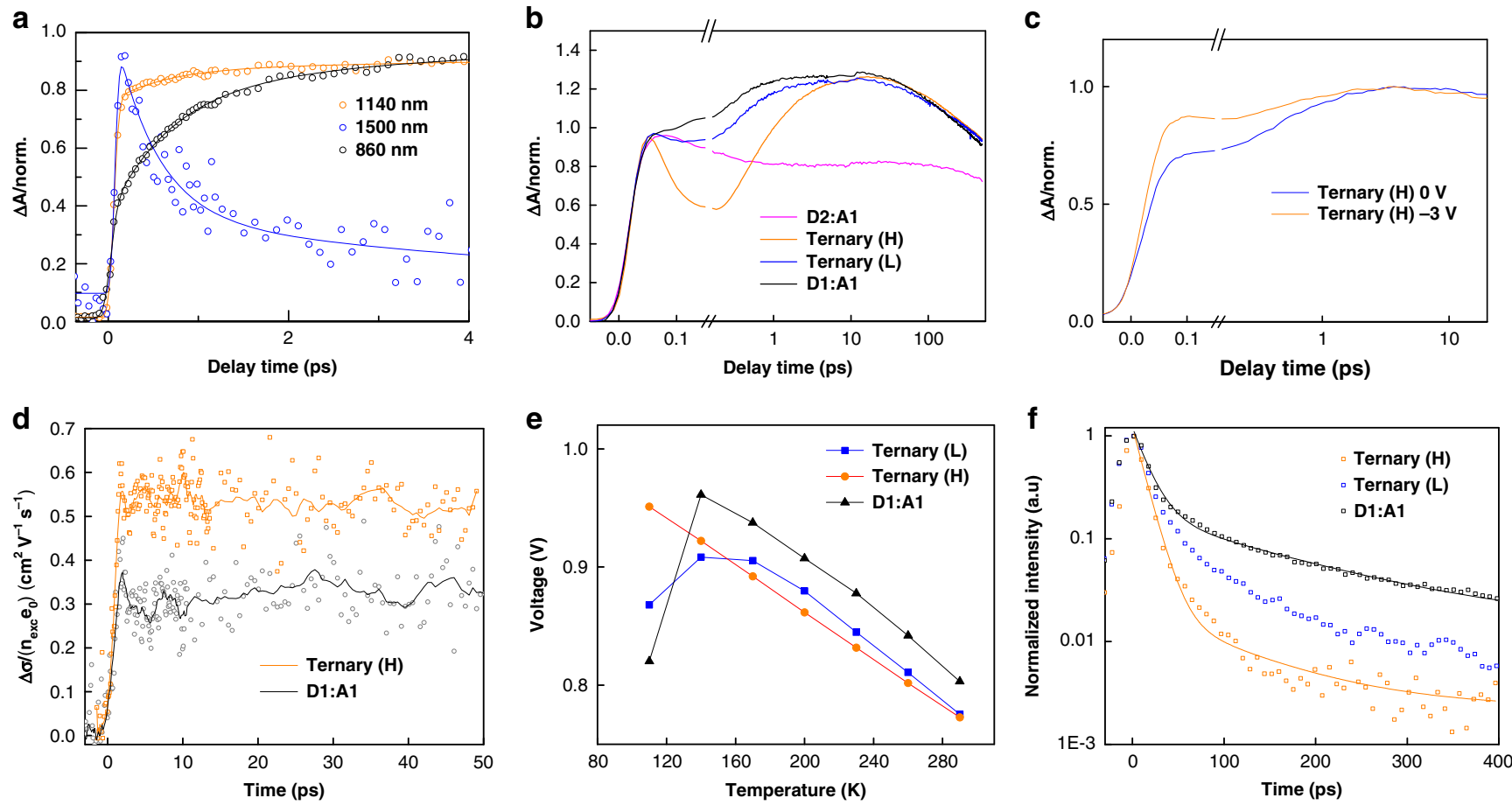

Fig. 2 Excited-state dynamics of blends. a Kinetics of Ternary(H), pumped at $700 \mathrm{~nm}$, probed at $860 \mathrm{~nm}, 1142 \mathrm{~nm}$ and $1500 \mathrm{~nm}$, respectively. $\mathbf{b}$ Kinetics of two ternary blends and two reference binary blends, normalized to the simultaneously acquired signal, pumped at $550 \mathrm{~nm}$ and probed at $860 \mathrm{~nm}$. c Transient absorption spectra under negative bias of Ternary $(\mathrm{H})$ device, pump at $700 \mathrm{~nm}$ and probe at $860 \mathrm{~nm}$. $\mathbf{d}$ Transient THz photoconductivity kinetics $\left(\lambda_{\mathrm{exc}}=400 \mathrm{~nm}, l_{\mathrm{exc}}=4.5 \times 10^{12} \mathrm{ph} / \mathrm{cm}^{2}\right)$. e Temperature dependent photovoltage evolution for three blend diodes. $\mathbf{f}$ Kinetic curves derived from the timeresolved photoluminescence data by integrating over the spectral range $740-950 \mathrm{~nm}$, pump at $700 \mathrm{~nm}$.

the photo-generated species in the $D 2: A 1$ blend has very low mobility that is below the detection limit. The mobility of $\sim 0.55$ $\mathrm{cm}^{2} /$ Vs estimated for the Ternary $(\mathrm{H})$ blend is higher than in the $D 1: A 1$ blend $\left(0.3 \mathrm{~cm}^{2} / \mathrm{Vs}\right)$, implying that the species present at this timescale (up to $50 \mathrm{ps}$ ) are relatively mobile and charged. The absence of photoconductivity signal in $D 2: A 1$, and the lower content of $D 1$ in Ternary $(\mathrm{H})$ compared to $D 1: A 1$ blend, are strong indicators that higher photoconductivity in the Ternary (H) blend arises from D1. Furthermore, this also means there should be an ultrafast charge generation preceding the time scale that TRTS can access $(<100 \mathrm{fs})$, resulting in the increased photoconductivity in the Ternary $(\mathrm{H})$ blend. These results are consistent with the TA kinetics and confirm that mobile charged species are generated through an ultrafast charge separation in the Ternary $(\mathrm{H})$ blend. Notice that the photoconductivity traces of $D 1: A 1$ and Ternary $(\mathrm{H})$ blend do not show any decay up to 50 ps. This also means that charge carriers can be efficiently extracted and collected as photocurrent, thus directly contributing to the enhanced performance of the Ternary $(\mathrm{H})$ device.

We have also studied the steady-state charge dynamics, following the temperature dependent $V_{\text {oc }}$ of the three different devices, as illustrated in Fig. 2e. Since all generated charge carriers recombine at $V_{\mathrm{oc}}$ and no long-range charge transport issue is involved, we measure the $V_{\mathrm{oc}}$ to exclusively focus on the influence of charge separation on the performance of the device. Here, the resulting charge generation is entropy controlled on the picosecond time scale ${ }^{19,20}$, which is consistent with timescale observed in our TRTS measurements. For the Ternary $(\mathrm{H})$ device, the $V_{\mathrm{oc}}$ keeps increasing with decreasing temperature (to $110 \mathrm{~K}$ ), which is significantly different from the other two devices. Note that the calculated charge carrier density shares the same tendency with the $V_{o c}$, as a function of temperature ${ }^{21,22}$. This linear feature indicates an efficient charge separation in the Ternary $(\mathrm{H})$ device, in agreement with our transient results.
A previous study has demonstrated that ultrafast charge separation at the donor/acceptor interface can be influenced by the fullerene aggregate size 23,24 . Our grazing-incidence smallangle X-ray scattering results (Supplementary Fig. 10) demonstrate that the fullerene aggregate size and order are similar in these three different blends. Furthermore, we also rule out the possibility that varied interfacial areas between donor and acceptor $^{25}$ contribute to the differences, as comparable interfacial areas are observed in the three devices (Supplementary Fig. 11).

Based on quantum theoretical modeling, it has been proposed that the dynamic disorder due to electronic-vibrational interactions always increases the charge separation barrier; in contrast, static disorder makes the high-energy CS states more accessible $^{26,27}$. We evaluate the static disorder by experiments using a detailed analysis of temperature dependent space-charge limited current (SCLC) (Supplementary Fig. 12). Based on the simulations, we find that the energetic disorder $\sigma$ value for holes is comparable in the three different devices (Supplementary Fig. 13).

These results demonstrate that the differences in morphology, interface area and disorder cannot explain the differences we have observed among the three blends, and therefore a novel mechanism must account for the ultrafast charge generation in the high-performance ternary blend.

Coherence in photocurrent generation. Similar to the high excitation energy $(550 \mathrm{~nm})$ results, there is an efficient exciton dissociation in the Ternary $(\mathrm{H})$ blend when we selectively excite D1 (700 nm) (Fig. 2f, Supplementary Fig. 9). Furthermore, as the rates of free charge generation (Supplementary Fig. 7) and bias dependence (Supplementary Fig. 8) have the same results as under $550 \mathrm{~nm}$ excitation, it is rational to assume a participation of the CS state in the free charge generation process under $700 \mathrm{~nm}$ excitation. This result indicates that the fast charge separation 
a

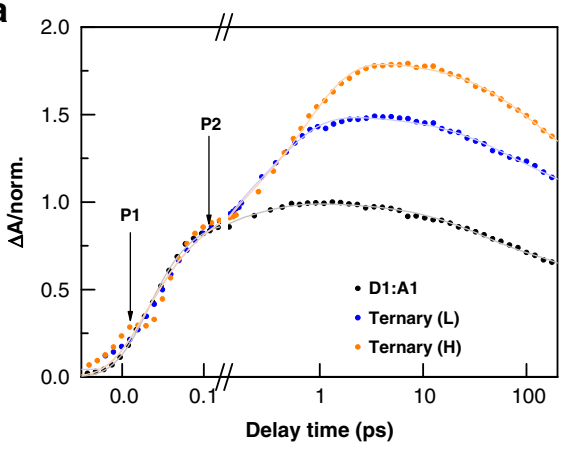

d

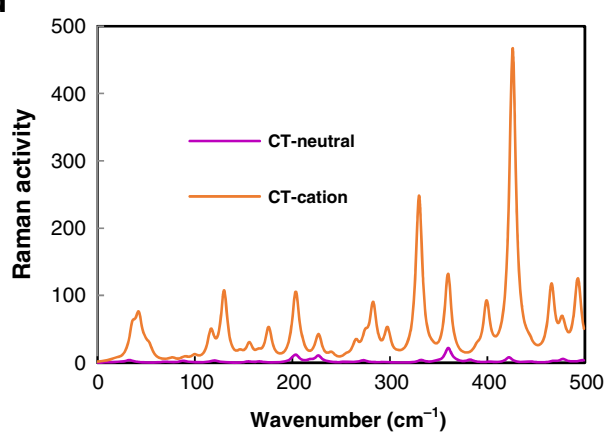

b

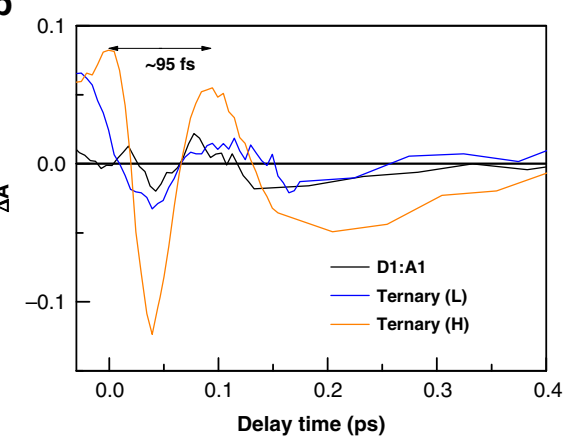

e

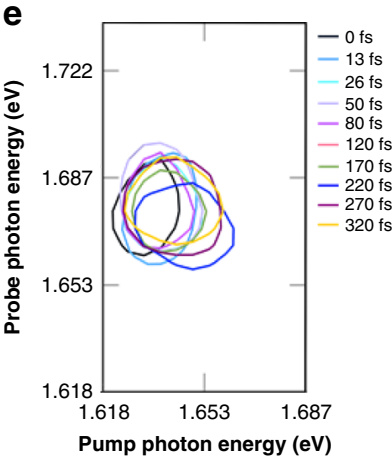

C

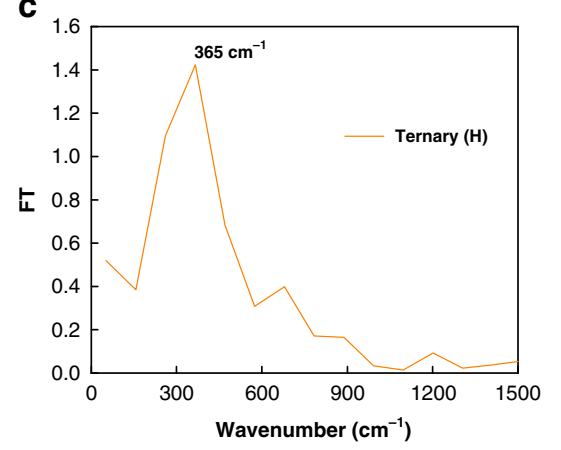

f

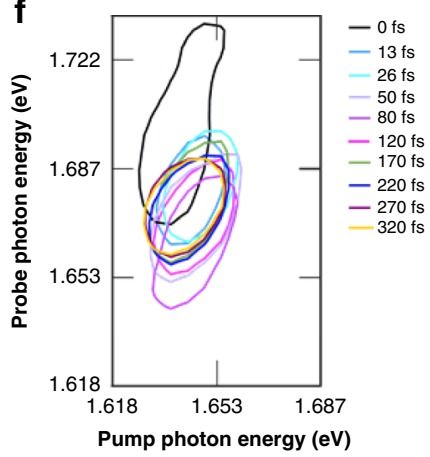

g

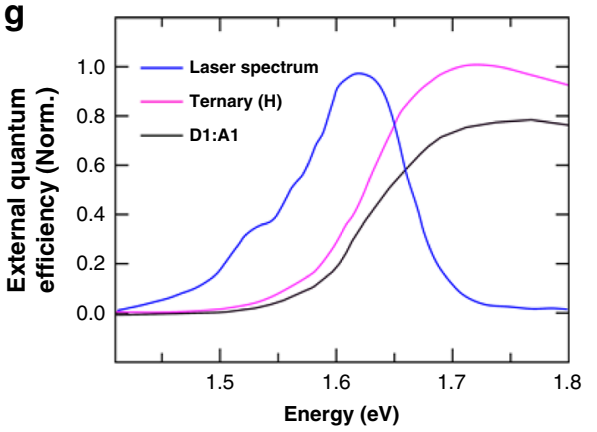

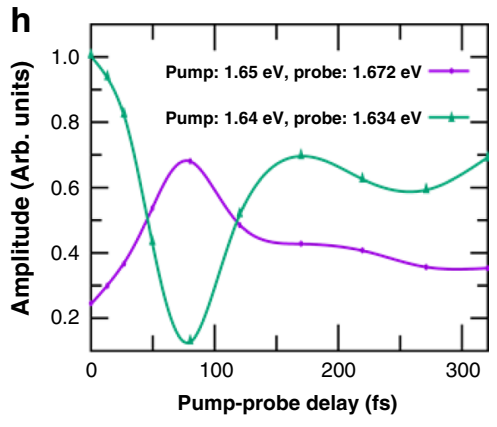

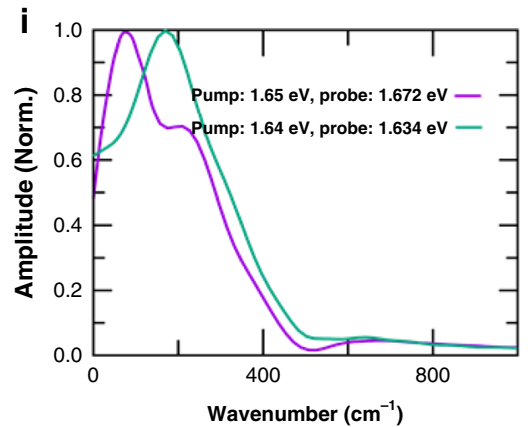

Fig. 3 Coherent dynamics of blends. a Kinetic process of the three blends, pumped at $700 \mathrm{~nm}$ and probed at $860 \mathrm{~nm}$, normalized at around $100 \mathrm{fs}$. The charge separation process occurred before $100 \mathrm{fs}$, followed by the charge generation process. The oscillation signal of Ternary $(\mathrm{H})$ as indicated by the arrow. b Corresponding oscillation signal. c Fourier transformed spectrum reveals the frequency of the oscillatory components at $365 \mathrm{~cm}^{-1}$. d Raman spectra calculated at the B3LYP/6-31+G(d) level of theory for the neutral and charged CT conformer. e A zoomed version of the 2DPS peak shifts (contour at $90 \%$ of the maximum) for the D1:A1 and $\mathbf{f}$ Ternary $(\mathrm{H})$ devices. The color indicates the time delay between the pump and the probe. $\mathbf{g}$ EQE of the two devices as a function of excitation energy, and the excitation spectrum of the laser. $\mathbf{h}$ Quantum beats observed at two selected positions in the 2DPS spectra of the Ternary $(\mathrm{H})$ device and $\mathbf{i}$ the corresponding Fourier transforms.

process is excitation-energy independent, unlike the case which involves molecular ordering and a dependency of the energetic landscape ${ }^{16}$.

In particular, the initial rise in the kinetics of the Ternary $(\mathrm{H})$ blend shows a pronounced oscillatory behavior (Fig. 3a), with an amplitude that is significantly higher than in the other two blends (Fig. 3b); these measurements have been done under the same excitation intensity. We have analyzed the oscillatory components by taking the Fourier transform after subtraction of the slowly varying background. The spectra shows a pronounced broad feature below $500 \mathrm{~cm}^{-1}$ encompassing a number of low frequency vibrational modes. The main peak at $\sim 365 \mathrm{~cm}^{-1}$ (Fig. 3c) is similar to the ground-state vibrational mode at $\sim 370 \mathrm{~cm}^{-1}$, detected by resonance Raman spectroscopy of the blends and materials (Supplementary Fig. 14). For the Ternary $(\mathrm{H})$ blend (Supplementary Fig. 7), a significantly increased charge carrier generation rate was observed after the initial $100 \mathrm{fs}$; this result is consistent with the charge transfer excitons kinetics (Fig. 3a), also demonstrating a significantly enhanced generation rate after the initial $100 \mathrm{fs}$, and indicates that the initial $100 \mathrm{fs}$ oscillatory feature is coupled with the ultrafast charge separation process. Furthermore, the oscillation persists $\sim 200 \mathrm{fs}$, longer than the ultrafast charge separation process. This result is different from the high frequency mode reported elsewhere ${ }^{9}$. The correlation between charge transfer excitons (and/or charge carrier) density and oscillation amplitude indicates that the excited-state vibrational mode with a frequency of $\sim 365 \mathrm{~cm}^{-1}$ drives the charge separation process. We have analyzed the vibrational mode in detail considering a model system of $D 1$ based on quantum theory (Fig. 3d, Supplementary Fig. 15). The main peak around $360 \mathrm{~cm}^{-1}$ is in agreement with the experimental results obtained from the Fourier transform of the TA and from the Raman spectra. The vibrational mode can be attributed mainly to an in-plane breathing of the benzo[1,2-b;4,5- $\left.\mathrm{b}^{\prime}\right]$ dithiophene donor unit in D1 (Fig. 4a; Supplementary Movie 1). 
a

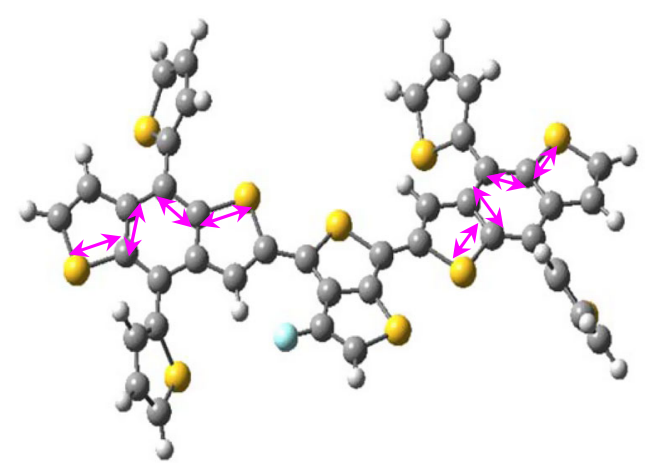

C

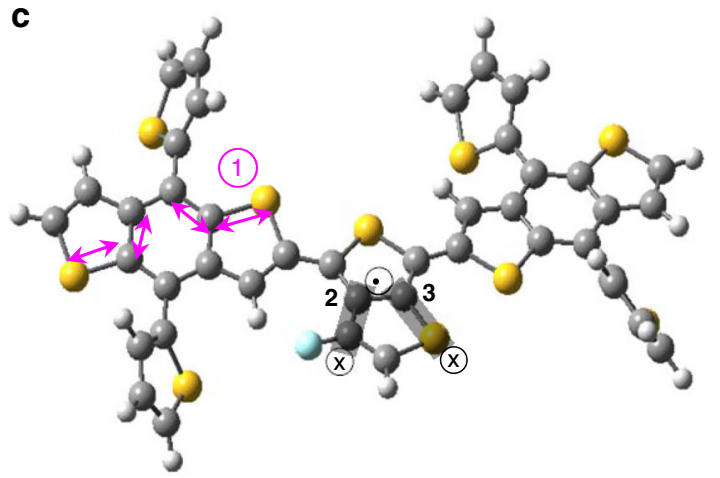

b

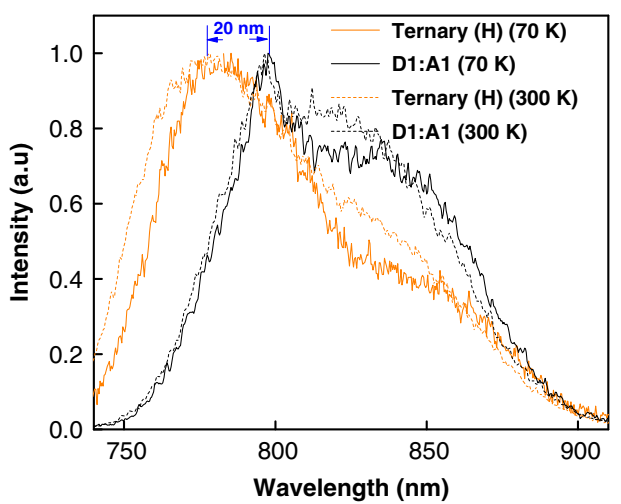

d
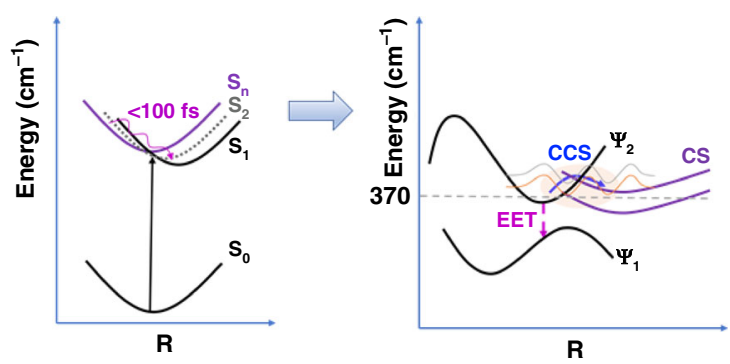

Fig. 4 Coherent kinetic process. a The in-plane breathing mode. The initial excitation delocalized by the in-plane breathing mode. Vibration direction indicated by arrow. $\mathbf{b}$ The transient photoluminescence peak of the Ternary $(\mathrm{H})$ demonstrates a blue shift compared to the D1:A1 blend at the first 20 ps timescale. c Out-of-plane mode (gray shadow). For the $200 \mathrm{~cm}^{-1}$ vibration mode, mainly due to the out-of-plane vibration of 3-fluorothieno[3,4-b] thiophene by the $C_{2}$ and $C_{3}$ bond. The in-plane breathing mode of the benzo[1,2-b;4,5-b'] dithiophene(1) simultaneously with the out-of-plane vibration of 3fluorothieno[3,4-b]thiophene, aid in charge separation simultaneously during the first $200 \mathrm{fs}$. Vibration direction indicated by arrow. $\mathbf{d}$ Kinetic approach for excitation energy transfer (EET) and coherent charge separation (CCS) process. Here, the resulting charge separated states (CS) are delocalized. Potential energy surfaces sections (PES) defined by the electronic wavefunctions $\left(\Psi_{1}, \Psi_{2}\right)$ of the excited states as a function of torsional, ring-breathing and intra/ intermolecular bond stretching coordinates $(R)$. The gray and pink line represent the EX and CS vibronic wavefunction, extended on both PES.

We have further analyzed the Raman activity corresponding to the in-plane breathing mode. Compared to the neutral, the positively charged CT conformer demonstrates a significant enhancement of the Raman activity (Fig. 3d, Supplementary Fig. 15). This result indicates the breathing mode is in resonance with the ultrafast charge transfer process, which results in efficient CS states formation, consistent with our experimental results. Thus, the initial excitation can be dissociated into CS states through this specific breathing mode.

Our transient results demonstrate that the ultrafast process from EX to CS is temperature independent (Supplementary Fig. 5), and the corresponding oscillation signal dephases quickly (in $<200 \mathrm{fs}$, consistent with the recently resolved electronic coherence lifetime $\left.{ }^{28}\right)$. Furthermore, the energy gap between the initial EX (emission peak $\sim 800 \mathrm{~nm}$ ) and the CS states (emission peak $\sim 780 \mathrm{~nm}$ ) (Fig. 4b, Supplementary Fig. 16), is resonant with the breathing mode $\left(\sim 365 \mathrm{~cm}^{-1}\right)$. Thus, the resulting electron-vibrational (vibronic) coupling, via electronic states coupled to the in-plane breathing mode, plays the most effective role in promoting CS formation when it is resonant with the transition energy ${ }^{29}$. These results further indicate that the electronic coherence may also contribute to the ultrafast charge separation in the Ternary $(\mathrm{H})$ blend. Note here that the product CS states are located at higher energy than the primary EX states. This uphill energy process thus utilizes coherence, breaking the energy barrier between these two states, which is unlike the normal downhill hopping case $\mathrm{s}^{30}$. Considering that the coherence process lives as long as the breathing period lasts, electronic coherence could be enhanced by this vibrational coherence.

In order to further conclusively prove that the coherent motion is involved in the charge transfer process and the subsequent photocurrent generation, we have used $2 \mathrm{D}$ coherent photocurrent spectroscopy (2DPS) ${ }^{31-33}$. This method is an extension of $2 \mathrm{D}$ electronic spectroscopy, but uses photocurrent generated in a device as the measured signal when illuminated by two pairs of pulses of light in a pump and probe setup for following the nonlinear response of the excited photodiode (see Methods section). In distinction to our method for nonlinear imaging of photocurrent for identifying recombination mechanisms ${ }^{34,35}$, where the amplitude of the nonlinear incoherent response is used, here the phase of the photocurrent is extracted by varying the interpulse time delays. This has been shown to account for the coherent nonlinear interactions in this type of spectroscopic measurement ${ }^{36,37}$. For all the investigated devices here, the same setup with a temporal resolution of about $15 \mathrm{fs}$ and identical experimental conditions have been used. The spectra at the zero time delay between the pump and probe shows that the main peak is shifted above the diagonal (Fig. 3e, f, Supplementary Fig. 17). There are different mechanisms, such as many body effects ${ }^{38}$ and AC (optical) Stark effects ${ }^{39}$ that cause the shifts. Among the two effects, the upshift in the probe frequency indicates that the AC Stark effect plays the dominant role. The AC Stark effect can lower as well as increase the band gap 
of the material depending on the excitation spectrum ${ }^{40}$. In our experiment, most of the excitation spectrum (Fig. 3g) is below the band gap for both the devices. In this case, the AC Stark shift increases the band gap, which is observed as a shift in the 2D peak above the diagonal. In order to further analyze the $2 \mathrm{D}$ spectra, we show the evolution of the peak position as contour lines at $90 \%$ of the maximum amplitude for the $D 1: A 1$ and Ternary $(\mathrm{H})$ devices in Fig. 3e, f, respectively. After the pump-probe overlap (i.e., beyond $15 \mathrm{fs}$ ), the main peak shifts to the diagonal. The peak position in both the samples is at $1.653 \mathrm{eV}(750 \mathrm{~nm})$, which corresponds to the excitonic transition. Note that the rise in the amplitude with energy is gradual compared to most of the semiconductor systems. This indicates that other bound states, such as polarons, also contribute to the photocurrent. Nevertheless, their contribution is insignificant compared to the excitons and excitations above the bandgap. Beyond the pulse overlap, we observe a significant oscillation of the peak position of the Ternary $(\mathrm{H})$ device within $200 \mathrm{fs}$. Then the peak position remains static. Such transient feature is not observed in the $D 1: A 1$ device (Fig. 3e). Compared to a pristine molecular system, the polymer blend is heterogeneous. A number of vibronic states from the different components in the blend contribute to the spectral features above the bandgap. As a consequence, the excitation above the bandgap results in a rather constant EQE without sharp spectral features (Fig. 3g), which makes it difficult to infer coherences as distinct off-diagonal peaks in the 2D spectra. This is similar to the continuum of states in a semiconductor. Nevertheless, the coherences can be inferred from the oscillations in the off-diagonal positions of the broad spectral lineshape. In order to quantitatively analyze the oscillation frequencies, we monitor the quantum beat signals at two positions on the off-diagonal as shown in Fig. $3 \mathrm{~h}$. In the first position, pump is at $1.65 \mathrm{eV}$ and probe is at $1.672 \mathrm{eV}$ and in the second position, pump is at $1.644 \mathrm{eV}$ and probe is at $1.634 \mathrm{eV}$. The oscillations in these two positions are in antiphase. The Fourier transforms show significant vibrational contribution to the photocurrent at frequencies below $500 \mathrm{~cm}^{-1}$ (Fig. 3i). The main peak in the spectra is around $200 \mathrm{~cm}^{-1}$, which is different from the main peak in the TA results. This result can be rationalized based on the fact that in the detected $2 \mathrm{D}$ photocurrent, the results are weighted by the EQE of the photocurrent, while in the TA we only measure the optical response. Note that the window of oscillation frequencies (Fig. 3i) includes the vibrational mode that is resolved by TA. Similar to the TA results, the oscillations dephase quickly $(\sim 200 \mathrm{fs})$. Our results show that the low frequency vibrational modes have greater contribution to the coherent photocurrent generation. The low vibrational mode $\left(\sim 200 \mathrm{~cm}^{-1}\right)$ can be mainly attributed to the out-of-plane vibration of the 3fluorothieno[3,4-b]thiophene donor unit in D1 (Fig. 4c, Supplementary Movie 2 and Movie 3). Such out-of-plane conformer hinders close main-chain stacking and influences the local configuration of the $A 1$ moieties near the $D 1$ backbone $e^{41,42 \text {, }}$ facilitates the intermolecular charge transport between $D 1$ and $A 1^{43}$. Note here that both vibrational modes $\left(\sim 365\right.$ and $\left.200 \mathrm{~cm}^{-1}\right)$ aid in charge separation simultaneously and persist in the first 200 fs. The results clearly indicate a coherent charge transfer process within $200 \mathrm{fs}$ of the excitation, that has a significant contribution to the photocurrent in the Ternary $(\mathrm{H})$ device.

It has been suggested that there is strong coupling between electronic potential energy and the torsional mode, which facilitates the initial coherent excitation energy transfer (EET) $(<100 \mathrm{fs})$ and charge separation process ${ }^{13,14}$. Transient results demonstrate that the simultaneous process of EET from the excited state is significant in the Ternary (L) and $D 1: A 1$ blend, compared to the Ternary $(\mathrm{H})$ blend (Supplementary Fig. 19). Due to efficient charge separation, the competing initial EET process is suppressed in the Ternary $(\mathrm{H})$ blend. Our study indicates that an initial planarization from a torsional mode, is not the first decisive step in this ternary blend.
Here, the coherent charge separation (CCS) outcompetes the electronic relaxation and results in CS products (Fig. 4d).

To generalize the contribution of the ultrafast charge separation in OPVs performance, we also study blends with a non-fullerene acceptor (Supplementary Fig. 20). ITIC has planar and rigid conformation and has been widely used in high efficiency donor/acceptor blends ${ }^{44}$. The rigidity suppresses torsional relaxation, and thus improves efficient EET process. TA demonstrates that, in the initial time scale (100 500 fs), EET is significant in both binary and ternary blends. Thus, the competing charge separation is suppressed, and resulting in similar device performance (Supplementary Fig. 21).

\section{Discussion}

The primary charge separation process in this organic photovoltaic blend occurs by vibronic coherence and is controlled by the electronic and vibrational coupling unique to the low frequency in-plane breathing mode and out-of-plane vibration mode. Our study shows that even if the coherence time is short $(\sim 200 \mathrm{fs})$, this process is helpful in reducing charge-pair Coulomb interaction and efficiently generating charged species, thereby contributing to the photocurrent enhancement. Specifically, the breathing modes found here can work as an effective design building block, giving clear inspiration for designing chemical structures that exploit coherent quantum mechanical effects in organic materials and nanostructures. Coherence phenomena have been proposed to contribute in some biological photosynthetic systems ${ }^{29,45-47}$, and some reviews have also proposed that coherence might enhance charge transfer ${ }^{46,48}$. Very recently, coherence was found to play a key role for the near-unity CS efficiency in a natural reaction center complex ${ }^{28}$. To explore the next-generation of photovoltaic materials, new design strategies based on understanding the working mechanism are needed. We anticipate that our identification of a dynamic contribution from vibronic coherence can be used to design new molecules and materials for improved charge generation.

\section{Methods}

Sample preparation. PTB7-th and ITIC were purchased from Solarmer Material Inc, Beijing, China. PCDTBT were purchased from 1-Material Inc, Canada. PCDTBT with low and high molecular weight is $\mathrm{Mw} 64 \mathrm{kDa}$ and $450 \mathrm{kDa}$, respectively.

Two common polymers PTB7-th (D1), (Poly[4,8-bis(5-(2-ethylhexyl)thiophen 2-yl)benzo[1,2-b;4,5-b'] dithiophene-2,6-diyl-alt-(4-(2-ethylhexyl)-3-fluorothieno [3,4-b]thiophene-)-2-carboxylate-2-6-diyl)]) and PCDTBT (D2), (poly[N11W-henicosanyl-2,7-carbazole-alt-5,5-(40, 70-di-2-thienyl-20, 10, 30benzothiadiazole)]), were used as electron donors. The $\mathrm{PC}_{71} \mathrm{BM}(\mathbf{A 1})$, (phenylC71-butyric acid methyl ester) and small molecule ITIC $^{44}$ (A2), (3,9-bis(2methylene-(3-(1,1-dicyanomethylene)-indanone))-5,5,11,11-tetrakis(4-

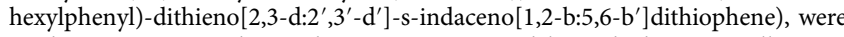
used as acceptors in this study. PCDTBT is a widely studied noncrystalline polymer, with a planar structure in the excited state ${ }^{49}$. When PCDTBT is blended with PCBM, internal quantum efficiency close to $100 \%$ was obtained ${ }^{49,50}$. By using different molecular weights and optimizing the donor phase loading ratios in the ternary D1:D2:A1 blend with respect to the binary blend, a substantial improvement in short-circuit current density $\left(J_{\mathrm{sc}}\right)$ and fill factor $(\mathrm{FF})$, which resulted in a substantially increased power conversion efficiency of $9.5 \%$. We focus on two optimized ternary blends (Ternary (L), D1:D2(L):A1 =9:1:10, with low molecular weight in $D 2$; Ternary $(\mathbf{H}), D 1: D 2(\mathrm{H}): A 1=7: 3: 10$, with high molecular weight in D2) and two reference binary blends (D1:A1, D2:A1) (Supplementary Fig. 1). Absorption and photoluminescence spectra, external quantum efficiency (EQE) and current $(J)$-voltage $(V)$ curves are provided in Fig. 1 of the Supplementary Information.

Binary and ternary devices were prepared in the normal device configuration ITO/PEDOT:PSS $(40 \mathrm{~nm}) /$ active layer $(100 \mathrm{~nm}) / \mathrm{LiF}(0.6 \mathrm{~nm}) / \mathrm{Al}(90 \mathrm{~nm})$. PEDOT: PSS (Baytron P VP Al 4083) was spun-cast and baked at $140{ }^{\circ} \mathrm{C}$. The active layer was spin-coated from a $10 \mathrm{mg} \mathrm{mL}^{-1}$ chlorobenzene solution. $\mathrm{LiF}(0.6 \mathrm{~nm})$ and $\mathrm{Al}$ $(100 \mathrm{~nm})$ were used as cathode, was thermally evaporated under vacuum $\left(<10^{-7}\right.$ torr). Hole only devices were prepared in the device configuration ITO/PEDOT: PSS/active layer/ $\mathrm{MoO}_{3} / \mathrm{Al}$.

For indication case, in the main and Supplementary Materials text, the optimized blends are Ternary (H) (PTB7-th:PCDTBT(H):PC ${ }_{71} B M(7: 3: 10)$, 
PCDTBT with high molecular weight) and Ternary (L) (PTB7-th:PCDTBT(L): $\mathrm{PC}_{71} \mathrm{BM}$ (9:1:10), PCDTBT with low molecular weight).

Low temperature measurement. Normal devices and single-carrier devices were mounted in a liquid-nitrogen cryostat for temperature-dependent measurements. The $J-V$ curves were measured using a Keithley 2400 source meter at temperatures ranging from 90 to $300 \mathrm{~K}$. The temperature was monitored and controlled using a LakeShore 330 Autotuning Temperature Controller. The light source was a blue laser $(532 \mathrm{~nm})$, and the light intensity was tuned to give a $V_{\mathrm{OC}}$ similar to one-sun conditions at room temperature for Voc-T test. Photoluminescence (PL) and electroluminescence (EL) spectra were detected using a light guide positioned close to the cryostat window. The emission detection system was a Newton EM-CCD Si array detector cooled at $-60^{\circ} \mathrm{C}$ in conjunction with a Shamrock sr 303i spectrograph from Andor Tech.

Time resolved transient absorption (TA) instrumentation. Transient absorption (near-infrared) measurements were performed by means of regeneratively amplified, mode-locked Yb:KGW (Ytterbium-doped potassium gadolinium tungstate) based femtosecond laser system (Pharos, Light conversion) operating at 1030 $\mathrm{nm}$ and delivering pulses of $200 \mathrm{fs}$ at a $1 \mathrm{kHz}$ repetition rate. This laser is then used to pump two non-collinearly phase-matched optical parametric amplifiers (NOPAs, Orpheus-N, Light Conversion). A first one was used to generate pump pulses centered at $540 \mathrm{~nm}$ with a pulse duration of $35 \mathrm{fs}$. The second NOPA was used to generate probe pulses of about $40 \mathrm{fs}$ duration in the spectral region from 850 to $960 \mathrm{~nm}$ for differential absorption measurements. The probe was time delayed with respect to the pump by a mechanical delay stage.

Transient absorption measurements (infrared) were performed using a Helios setup. The transient dynamics in fs-ns time region ( $50 \mathrm{fs}-7 \mathrm{~ns}$ ) was acquired by Helios that works in a nondegenerate pump-probe configuration. The pump pulses were generated from an optical parametric amplifier (OPerA Solo) that was pumped by a $1-\mathrm{kHz}$ regenerative amplifier (Coherent Libra, $800 \mathrm{~nm}, 50 \mathrm{fs}, 4 \mathrm{~mJ}$ ). A mode lock Ti-sapphire oscillator (Coherent Vitesse, $80 \mathrm{MHz}$ ) was used to seed the amplifier. For $800 \mathrm{~nm}$ pump, the laser from the regenerative amplifier was directly used. The probe pulses were a white light continuum generated by passing the $800 \mathrm{~nm}$ fs pulses through a $1 \mathrm{~cm}$ sapphire plate for the infrared part $(840-1600 \mathrm{~nm})$.

The measured sample solution was spin coated on the quartz substrates and mounted in a nitrogen cryostat. A consistency results of the near-infrared signal was achieved from the two different transient absorption setups.

Time resolved terahertz (THz) instrumentation. The photoconductivity kinetics were measured by a setup similar to that in the ref. ${ }^{2}$. Laser pulses $(796 \mathrm{~nm}, 80 \mathrm{fs}$ pulse length, $1 \mathrm{kHz}$ repetition rate) were generated by a regenerative amplifier (Spitfire Pro XP, Spectra Physics) seeded by a femtosecond oscillator (MaiTai, Spectra Physics). The laser beam was split into three. The first beam $(400 \mu \mathrm{J} / \mathrm{pulse})$ was used to generate $\mathrm{THz}$ radiation by optical rectification in a $\mathrm{MgO}: \mathrm{LiNbO}_{3}$ crystal. The second beam was used for electro-optical sampling of the THz pulses in a (110) ZnTe crystal. The third beam, which was used for excitation was converted to the second harmonic $(398 \mathrm{~nm})$ in a BBO crystal. To avoid absorption of the THz radiation by water vapor, the setup was purged with dry nitrogen. For all the investigated films in the text, the same time-resolved THz spectroscopy (TRTS) setup and identical experimental conditions were used.

The primary result of TRTS is the change in conductivity $(\Delta \sigma)$ of the material ${ }^{51}$ following optical excitation as expressed by the equation:

$$
\Delta \sigma=\xi \times\left(\mu_{\mathrm{e}}+\mu_{\mathrm{h}}\right)=-\frac{\Delta E_{\mathrm{exc}}(\omega)}{\Delta E_{\mathrm{gx}}(\omega)} \frac{c \varepsilon_{0}}{\mathrm{Fe}_{0}} \frac{1}{1-e^{-\alpha L}},
$$

where $\xi$ is quantum yield of charge generation, $\mu_{\mathrm{e}}$ and $\mu_{\mathrm{h}}$ are the electron and hole mobility, respectively, $\Delta E_{\text {exc }}$ is the THz electric field transmitted through the sample after photo excitation while $\Delta E_{\mathrm{gs}}$ is the ground state $\mathrm{THz}$ electric field, $\varepsilon_{0}$ is permittivity of vacuum, $c$ is velocity of light, $F$ is the fluence in $\mathrm{ph} \mathrm{cm}^{-2}, e_{0}$ is the elementary charge, $\alpha$ is the absorption coefficient, and $L$ the thickness of the sample. The quantity that is obtained from the above equation has the unit of mobility in $\mathrm{cm}^{2} \mathrm{~V}^{-1} \mathrm{~s}^{-1}$.

2D photocurrent spectroscopy. The schematic of the experimental setup is shown in the Supplementary Fig. 17. In the setup, a chirp pre-compensated pulsed laser beam (about $70 \mathrm{MHz}$ repetition rate, $15 \mathrm{fs}$ pulse duration and center wavelength of $790 \mathrm{~nm}$ ) from a Ti-Sapphire oscillator (Synergy from Femtolasers) is split into two. Each beam goes through a Mach-Zehnder interferometer (MZ). Acoustooptic modulators, AOM 1,2,3, and 4 on the arms of the interferometers MZ1 and MZ2 modulate the phases of the beams at frequencies $\phi_{1}, \phi_{2}, \phi_{3}$, and $\phi_{4}$, respectively. The delay line DL1 controls the time delay $\tau$ between the first two pulses (pulse 1 and pulse 2). Similarly, DL3 controls the time delay $t$ between the last two pulses (pulse 3 and pluse 4). The delay $T$ between pulse 2 and pulse 3 is controlled by DL2. All the beams after MZ1 and MZ2 are recombined using a 50/50 beam splitter. One of the outputs from the beam splitter is used to excite the sample $\mathrm{S}$, while the other output goes to a monochromator MC. The monochromator selects a narrow spectrum of the light, which provides the reference detected by a Siphotodiode. The photocurrent signal from the sample is amplified by a preamplifier (SR570, Stanford Research Systems). The signal and the reference are digitized simultaneously at the rate of $10 \mathrm{MSa} / \mathrm{s}$ (mega samples per second). The phases and amplitudes of the signals modulated at the frequencies $\phi_{4}-\phi_{3}-\phi_{2}+$ $\phi_{1}$ and $\phi_{4}-\phi_{3}+\phi_{2}-\phi_{1}$ are extracted from the digitized data by using the algorithms of the generalized lock-in amplifier (GLIA) ${ }^{52-54}$. The measurements are done in time domain by varying the time delays. The spectra in the frequency domain are obtained by the Fourier transforms along $\tau$ and $t$ for each time delay of $T$. The Fourier transform along $\tau$ gives the pump frequency and the Fourier transform along $t$ gives the probe frequency for the different waiting times (population times) $T$. The real part of the 2D spectra obtained from the signals at the two frequencies are summed to obtain the total correlation spectra, which are shown in Supplementary Fig. 17.

Vibrational modes calculation based on quantum theory. Methodology: Optimization and Raman spectra has been calculated at the B3LYP/6-31+ G(d) level of theory using the Gaussian Package.

Cis trans conformation between the small and large units. Model systems: To investigate the conformational space between the large and the small unit of PTB7th, we build the four following models comprising a small unit between two large units in combination of cis and trans position.

\section{Data availability}

The datasets generated during and/or analysed during the current study are available from the corresponding author on reasonable request.

Received: 10 June 2019; Accepted: 9 January 2020;

Published online: 30 January 2020

\section{References}

1. Caruso, D. \& Troisi, A. Long-range exciton dissociation in organic solar cells. Proc. Natl Acad. Sci. 109, 13498-13502 (2012).

2. Liu, J. et al. Fast charge separation in a non-fullerene organic solar cell with a small driving force. Nat. Energy 1, 16089 (2016).

3. Bakulin, A. A. et al. The role of driving energy and delocalized states for charge separation in organic semiconductors. Science 335, 1340-1344 (2012).

4. Deibel, C., Strobel, T. \& Dyakonov, V. Origin of the efficient polaron-pair dissociation in polymer-fullerene blends. Phys. Rev. Lett. 103, 036402 (2009).

5. Jailaubekov, A. E. et al. Hot charge-transfer excitons set the time limit for charge separation at donor/acceptor interfaces in organic photovoltaics. Nat. Mater. 12, 66-73 (2013).

6. Grancini, G. et al. Hot exciton dissociation in polymer solar cells. Nat. Mater 12, 29-33 (2013).

7. Brabec, C. J. et al. Tracing photoinduced electron transfer process in conjugated polymer/fullerene bulk heterojunctions in real time. Chem. Phys. Lett. 340, 232-236 (2001)

8. Cooke, D. G., Krebs, F. C. \& Jepsen, P. U. Direct observation of sub-100 fs mobile charge generation in a polymer-fullerene film. Phys. Rev. Lett. 108, 056603 (2012).

9. Falke, S. M. et al. Coherent ultrafast charge transfer in an organic photovoltaic blend. Science 344, 1001-1005 (2014).

10. De Sio, A. et al. Tracking the coherent generation of polaron pairs in conjugated polymers. Nat. Commun. 7, 13742 (2016).

11. Vella, E. et al. Ultrafast decoherence dynamics govern photocarrier generation efficiencies in polymer solar cells. Sci. Rep. 6, 29437 (2016).

12. Collini, E. \& Scholes, G. D. Coherent intrachain energy migration in a conjugated polymer at room temperature. Science 323, 369-373 (2009).

13. Nelson, T. R. et al. Coherent exciton-vibrational dynamics and energy transfer in conjugated organics. Nat. Commun. 9, 2316 (2018).

14. Delor, M. et al. Toward control of electron transfer in donor-acceptor molecules by bond-specific infrared excitation. Science 346, 1492-1495 (2014).

15. Paquin, F. et al. Two-dimensional spatial coherence of excitons in semicrystalline polymeric semiconductors: effect of molecular weight. Phys. Rev. B 88, 155202 (2013).

16. Jakowetz, A. C. et al. Visualizing excitations at buried heterojunctions in organic semiconductor blends. Nat. Mater. 16, 551 (2017).

17. Sampaio, R. N. et al. Kinetics teach that electronic coupling lowers the freeenergy change that accompanies electron transfer. Proc. Natl Acad. Sci. 115, 7248-7253 (2018).

18. Ponseca, C. S. Jr et al. Ultrafast terahertz photoconductivity of bulk heterojunction materials reveals high carrier mobility up to nanosecond time scale. J. Am. Chem. Soc. 134, 11836-11839 (2012).

19. Melianas, A. et al. Photo-generated carriers lose energy during extraction from polymer-fullerene solar cells. Nat. Commun. 6, 8778 (2015). 
20. Van Eersel, H., Janssen, R. A. J. \& Kemerink, M. Mechanism for efficient photoinduced charge separation at disordered organic heterointerfaces. Adv. Funct. Mater. 22, 2700-2708 (2012).

21. Gao, F., Tress, W., Wang, J. \& Inganäs, O. Temperature dependence of charge carrier generation in organic photovoltaics. Phys. Rev. Lett. 114, 128701 (2015).

22. Rauh, D., Wagenpfahl, A., Deibel, C., \& Dyakonov, V. Relation of open circuit voltage to charge carrier density in organic bulk heterojunction solar cells. Appl. Phys. Lett. 98, 69 (2011).

23. Savoie, B. M. et al. Unequal partnership: asymmetric roles of polymeric donor and fullerene acceptor in generating free charge. J. Am. Chem. Soc. 136, 2876-2884 (2014).

24. Gélinas, S. et al. Ultrafast long-range charge separation in organic semiconductor photovoltaic diodes. Science 343, 512-515 (2014).

25. Vandewal, K. et al. Increased open-circuit voltage of organic solar cells by reduced donor-acceptor interface area. Adv. Mater. 26, 3839-3843 (2014).

26. Tummala, N. R., Zheng, Z., Aziz, S. G., Coropceanu, V. \& Bredas, J. L. Static and dynamic energetic disorders in the C60, PC61BM, C70, and PC71BM fullerenes. J. Phys. Chem. Lett. 6, 3657-3662 (2015).

27. Yan, Y., Song, L. \& Shi, Q. Understanding the free energy barrier and multiple timescale dynamics of charge separation in organic photovoltaic cells. J. Chem. Phys. 148, 084109 (2018).

28. Ma, F., Romero, E., Jones, M. R., Novoderezhkin, V. I. \& Van Grondelle, R. Both electronic and vibrational coherences are involved in primary electron transfer in bacterial reaction center. Nat. Commun. 10, 933 (2019).

29. Scholes, G. D. et al. Using coherence to enhance function in chemical and biophysical systems. Nature 543, 647-656 (2017).

30. Duan, H. G. et al. Nature does not rely on long-lived electronic quantum coherence for photosynthetic energy transfer. Proc. Natl Acad. Sci. 114, 8493-8498 (2017).

31. Karki, K. J. et al. Coherent two-dimensional photocurrent spectroscopy in a PbS quantum dot photocell. Nat. Commun. 5, 5869 (2014).

32. Bakulin, A. A., Silva, C. \& Vella, E. Ultrafast spectroscopy with photocurrent detection: watching excitonic optoelectronic systems at work. J. Phys. Chem. Lett. 7, 250-258 (2016).

33. Li, H. et al. Probing polaron excitation spectra in organic semiconductors by photoinduced-absorption-detected two-dimensional coherent spectroscopy. Chem. Phys. 481, 281-286 (2016).

34. Bergqvist, J. et al. New method for lateral mapping of bimolecular recombination in thin-film organic solar cells. Prog. Photovolt. 24, 1096-1108 (2016).

35. Kumar, P., Shi, Q. \& Karki, K. J. Enhanced radiative recombination of excitons and free charges due to local deformations in the band structure of MAPbBr3 perovskite crystals. J. Phys. Chem. C 123, 13444-13450 (2019).

36. Damtie, F. A., Wacker, A., Pullerits, T. \& Karki, K. J. Two-dimensional action spectroscopy of excitonic systems: explicit simulation using a phasemodulation technique. Phys. Rev. A 96, 053830 (2017).

37. Kalaee, A. A. S., Damtie, F. \& Karki, K. J. Differentiation of true nonlinear and incoherent mixing of linear signals in action-detected 2D spectroscopy. J. Phys. Chem. A 123, 4119-4124 (2019).

38. Chemla, D. S. Jagdeep Shah many-body and correlation effects in semiconductors. Nature 411, 549 (2001).

39. Becker, P. C., Fork, R. L., Brito Cruz, C. H., Gordon, J. P. \& Shank, C. V. Optical Stark effect in organic dyes probed with optical pulses of 6-fs duration. Phys. Rev. Lett. 60, 2462-2464 (1988).

40. Grynberg, G., Alain, A. \& Claude, F. Introduction to Quantum Optics: From the Semi-classical Approach to Quantized Light. (Cambridge University Press, Cambridge, 2010).

41. Graham, K. R. et al. Importance of the donor:fullerene intermolecular arrangement for high-efficiency organic photovoltaics. J. Am. Chem. Soc. 136, 9608-9618 (2014).

42. Tamura, H., Ramon, J. G., Bittner, E. R. \& Burghardt, I. Phonon-driven ultrafast exciton dissociation at donor-acceptor polymer heterojunctions. Phys. Rev. Lett. 100, 107402 (2008).

43. Bittner, E. R. \& Silva, C. Noise-induced quantum coherence drives photocarrier generation dynamics at polymeric semiconductor heterojunctions. Nat. Commun. 5, 3119 (2014).

44. Lin, Y. et al. An electron acceptor challenging fullerenes for efficient polymer solar cells. Adv. Mater. 27, 1170-1174 (2015)

45. Romero, E. et al. Quantum coherence in photosynthesis for efficient solar energy conversion. Nat. Phys. 10, 676-682 (2014).

46. Bredas, J. L., Sargent, E. H. \& Scholes, G. D. Photovoltaic concepts inspired by coherence effects in photosynthetic systems. Nat. Mater. 16, 35-44 (2016).
47. Karki, K. J. et al. Before Förster. Initial excitation in photosynthetic light harvesting. Chem. Sci. 10, 7923-7928 (2019).

48. Rafiq, S. \& Scholes, G. D. From fundamental theories to quantum coherences in electron transfer. J. Am. Chem. Soc. 141, 708-722 (2019).

49. Banerji, N., Cowan, S., Leclerc, M., Vauthey, E. \& Heeger, A. J. Exciton formation, relaxation, and decay in PCDTBT. J. Am. Chem. Soc. 132, 17459-17470 (2010).

50. Park, S. H. et al. Bulk heterojunction solar cells with internal quantum efficiency approaching 100\%. Nat. Photon 3, 297-302 (2009).

51. Ponseca, C. S. Jr \& Sundstrom, V. Revealing the ultrafast charge carrier dynamics in organo metal halide perovskite solar cell materials using time resolved THz spectroscopy. Nanoscale 8, 6249-6257 (2016).

52. Fu, S. et al. Generalized lock-in amplifier for precision measurement of high frequency signals. Rev. Sci. Instrum. 84, 115101 (2013).

53. Karki, K., Torbjörnsson, M., Widom, J. R., Marcus, A. H. \& Pullerits, T. Digital cavities and their potential applications. J. Instrum. 8, T05005-T05005 (2013).

54. Jin, A. et al. Note: High precision measurements using high frequency gigahertz signals. Rev. Sci. Instrum. 85, 126102 (2014).

\section{Acknowledgements}

We thank Dr. Wei Ma for performing grazing-incidence small-angle X-ray scattering measurements. This work has been supported by the Knut and Alice Wallenberg Foundation (KAW) through a Wallenberg Scholar grant to O.I. A.Y. acknowledges support of the KAW and Crafoord Foundations. W.M.C. thanks the Knut and Alice Wallenberg Foundation for support (KAW 2014.0041). Q.B. acknowledges the China Scholarship Council (CSC)(no.201508320244). Open access funding provided by Linköping University.

\section{Author contributions}

Q.B. and O.I. designed the project. Q.B. performed the device-based tests and participated in all experiments. F.M. and Q.W. carried out the transient absorption measurements. S.C. carried out the transient photoluminescence measurements. X.S. carried out the transient terahertz measurements. K.J.K. carried out the $2 \mathrm{D}$ photocurrent measurements and analysis. M.L. carried out the DFT calculations. I.B., W.C., C.P. and A.Y. participated in data interpretation. Q.B. analyzed the data and wrote the manuscript together with O.I. All authors discussed the results and commented on the final manuscript.

\section{Competing interests}

The authors declare no competing interests.

\section{Additional information}

Supplementary information is available for this paper at https://doi.org/10.1038/s41467 020-14476-w.

Correspondence and requests for materials should be addressed to Q.B. or O.I.

Peer review information Nature Communications thanks Christoph Lienau, Serge Tretiak and the other, anonymous, reviewer(s) for their contribution to the peer review of this work. Peer reviewer reports are available.

Reprints and permission information is available at http://www.nature.com/reprints

Publisher's note Springer Nature remains neutral with regard to jurisdictional claims in published maps and institutional affiliations.

Open Access This article is licensed under a Creative Commons Attribution 4.0 International License, which permits use, sharing, adaptation, distribution and reproduction in any medium or format, as long as you give appropriate credit to the original author(s) and the source, provide a link to the Creative Commons license, and indicate if changes were made. The images or other third party material in this article are included in the article's Creative Commons license, unless indicated otherwise in a credit line to the material. If material is not included in the article's Creative Commons license and your intended use is not permitted by statutory regulation or exceeds the permitted use, you will need to obtain permission directly from the copyright holder. To view a copy of this license, visit http://creativecommons.org/ licenses/by/4.0/

(C) The Author(s) 2020 